\title{
Experience with DPP-4 inhibitors in the management of patients with type 2 diabetes fasting during Ramadan
}

This article was published in the following Dove Press journal:

Vascular Health and Risk Management

24 December 2013

Number of times this article has been viewed

\section{Anja Schweizer' \\ Serge Halimi ${ }^{2,3}$ \\ Sylvie Dejager ${ }^{4}$}

'Novartis Pharma AG, Basel, Switzerland; ${ }^{2}$ Department of Diabetology, Endocrinology and Nutrition, University Hospital of Grenoble, France; 3joseph Fourier University, Grenoble, France; ${ }^{4}$ Novartis Pharma SAS, Rueil-Malmaison, France
Correspondence: Anja Schweizer Novartis Pharma AG, Postfach, $\mathrm{CH}-4002$ Basel, Switzerland

$\mathrm{Tel}+4$ I 6I 3243530

Fax +4I 6I 3247105

Email anja.schweizer@novartis.com
Abstract: A large proportion of Muslim patients with type 2 diabetes mellitus (T2DM) elect to fast during the holy month of Ramadan. For these patients hypo- and hyperglycemia constitute two major complications associated with the profound changes in food pattern during the Ramadan fast, and efficacious treatment options with a low risk of hypoglycemia are therefore needed to manage their T2DM as effectively and safely as possible. Dipeptidyl peptidase-4 (DPP-4) inhibitors modulate insulin and glucagon secretion in a glucose-dependent manner, and consequently a low propensity of hypoglycemia has consistently been reported across different patient populations with these agents. Promising data with DPP-4 inhibitors have now also started to emerge in patients with T2DM fasting during Ramadan. The objective of this review is to provide a comprehensive overview of the currently available evidence and potential role of DPP-4 inhibitors in the management of patients with T2DM fasting during Ramadan whose diabetes is treated with oral antidiabetic drugs, and to discuss the mechanistic basis for their beneficial effects in this setting.

Keywords: dipeptidyl peptidase-4, incretin, type 2 diabetes mellitus, hypoglycemia

\section{Introduction}

Based on a 2010 demographic study, the worldwide Muslim population is estimated to comprise approximately 1.6 billion people, representing about $23 \%$ of the world population. ${ }^{1}$ Approximately $60 \%$ of the global Muslim population in 2010 lived in Asia-Pacific and about $20 \%$ in the Middle East and North Africa. ${ }^{1}$ The prevalence of type 2 diabetes mellitus (T2DM) keeps growing rapidly, amounting to 371 million patients in 2012 worldwide, and has been steeply rising, particularly in those regions of the world with large Muslim populations. ${ }^{2}$ Ramadan is the ninth month of the Islamic calendar and generally lasts for about 28-30 days. Fasting during the month of Ramadan (Sawm) is one of the five main pillars in Islam. It forms an integral part of faith and is obligatory for all healthy adult Muslims. Fasting requires abstinence from food and fluid intake, use of oral medications and smoking from predawn to after sunset, while there are no restrictions on eating and drinking between sunset and dawn. Consequently, most Muslims eat two meals per day during the month of Ramadan, one meal after sunset (referred to as Iftar) and another usually smaller meal before dawn (referred to as Suhur). Often the consumption of sweets increases during Ramadan and Iftar, the main meal breaking the fast, is also a festive time, a time to share an important familial and social event.

Religious fasting is not intended to create excessive hardship and the Qur'an specifically exempts the ill person from fasting. While patients with diabetes are usually 
also advised not to fast from a medical point of view, many patients nevertheless insist on fasting for religious reasons as well as for social and cultural reasons. In the populationbased Epidemiology of Diabetes and Ramadan (EPIDIAR) study $\sim 3 \%$ of patients with type 1 diabetes mellitus (T1DM) and $~ 79 \%$ of patients with T2DM fasted for at least 15 days during Ramadan, ${ }^{3}$ and thus, the estimated number of patients with diabetes who fast during the month of Ramadan is $>50$ million worldwide. ${ }^{4}$

Ramadan implies major changes in dietary habits (with marked deviations from the usual amount and pattern of food and fluid intake), daily physical activities and sleeping patterns and the main metabolic risks for patients with T2DM associated with Ramadan include hypoglycemia, hyperglycemia, diabetic ketoacidosis, dehydration, and thrombosis. ${ }^{4}$ Among those, hypoglycemia (and to a lesser extent hyperglycemia) is the most frequent and serious complication. ${ }^{5}$

While variable trends for the change in glycemic control have been reported in different trials, the large retrospective EPIDIAR study reported a fivefold increase in hyperglycemic episodes requiring hospitalization during Ramadan compared with other months. ${ }^{3}$ In general, an adjustment of antidiabetic medications before Ramadan including marked dose reductions, and/or an increase in food/sugar intake when the fast is broken can underlie hyperglycemia during Ramadan. In addition, a long fasting period in the absence of adequate insulin can lead to excessive hepatic glycogen breakdown and increased gluconeogenesis, contributing to the development of hyperglycemia. ${ }^{4,6,7}$

Hypoglycemia is a frequent issue and concern in patients with T2DM as a whole ${ }^{8}$ and becomes a key concern in type 2 diabetic patients fasting during Ramadan. ${ }^{9}$ For example, the above mentioned EPIDIAR study showed a $7.5 \times$ increase in severe hypoglycemia (leading to hospitalization) during the month of Ramadan versus previous months. ${ }^{3}$ Furthermore, a prospective cohort study reported a risk ratio for hypoglycemia of 1.60 (95\% confidence interval [CI], 1.05-2.43) for Muslim diabetic patients during Ramadan fasting versus an equivalent non-fasting period, with good metabolic control (glycosylated hemoglobin $\left[\mathrm{HbA}_{1 \mathrm{c}}\right]<8 \%$ ) and older age ( $>60$ years) increasing the risk ratio more than twice. ${ }^{10}$ Decreased food intake or missed meals as well as irregular eating habits are well established risk factors for hypoglycemia, and the risk of hypoglycemia can be aggravated further by the choice of antidiabetic agents and occurs most frequently with medications that raise insulin levels independently of blood glucose, such as the oral insulin secretagogues (sulfonylureas [SUs] and glinides) or exogenous insulin. ${ }^{11}$ Therefore, these agents should be used with caution and be individualized in patients fasting during Ramadan. ${ }^{4}$ Among the SUs, gliclazide (immediate release or modified release), glimepiride, or glipizide are generally reported to have a lower incidence of hypoglycemia than glibenclamide/glyburide or chlorpropamide, with gliclazide often having the lowest incidence both in the general T2DM population ${ }^{12,13}$ and in patients with T2DM fasting during Ramadan. ${ }^{4,14}$

The various recommendations for the management of patients with T2DM who choose to fast during Ramadan include a detailed pre-Ramadan medical assessment, any necessary adjustment to medications (doses and/or timing changes), patient education, nutritional advice, and regular blood glucose monitoring. ${ }^{4,9,15-18}$

Since the timing of Ramadan follows the Islamic lunar calendar, which is 11-12 days shorter than the solar year and contains no intercalation, the period of the month of Ramadan varies each year and migrates throughout the seasons. As the hours of daylight depend both on the season as well as the geographical location, the duration of the fast can vary considerably between different years and countries. In the upcoming years, Ramadan will occur in the summer months in the northern hemisphere, thus many people will have to face increasingly prolonged fasting hours, in particular in regions far away from the equator (more than 18 hours a day). This further exacerbates the earlier described risk of hypoglycemia, and the individualized choice of drug treatment becomes even more important.

The class of dipeptidyl peptidase-4 (DPP-4) inhibitors works by blocking the degradation of endogenous glucagonlike peptide-1 (GLP-1) and glucose-dependent insulinotropic polypeptide (GIP), thus, extending their actions. The resulting increase in $\alpha$ - and $\beta$-cell sensitivity to glucose leads to glucose-dependent modulation of insulin and glucagon secretion. ${ }^{19}$ Consequently, DPP-4 inhibitors improve glycemic control with a low risk of hypoglycemia. ${ }^{20}$

Data with DPP-4 inhibitors in T2DM patients fasting during Ramadan have recently started to emerge, and given the present unmet medical need for this patient population, it is of considerable interest to systematically look at all the currently available evidence with these newer agents. Literature for consideration for the present review was obtained primarily through a search of PubMed to identify all English language articles relevant to the use of DPP-4 inhibitors during Ramadan. This review discusses the experience with DPP-4 inhibitors in patients with T2DM fasting during Ramadan whose diabetes is treated with oral 
antidiabetic drugs (OADs), and also provides mechanistic insight underlying their hypoglycemia profile.

\section{Experience with DPP-4 inhibitors while fasting during Ramadan}

Virtually no data with the new class of DPP-4 inhibitors during periods of fasting with respect to either tolerability or efficacy existed when the updated recommendations for management of diabetes during Ramadan were published in $2010,{ }^{4}$ and therefore their place could not be truly assessed. Since then, specific studies with DPP-4 inhibitors in patients with T2DM fasting during Ramadan, whose diabetes is treated with OADs, have been published. A systematic search of PubMed, complemented with a search on clinicaltrial. gov for ongoing trials, retrieved data with two of the DPP-4 inhibitors, ie, sitagliptin and vildagliptin.

\section{Sitagliptin}

The first of two studies which assessed sitagliptin in patients with T2DM fasting during Ramadan was a multicenter, randomized, open-label, pragmatic trial that enrolled 1066 patients from six Middle East countries (Egypt, Israel, Jordan, Lebanon, Saudi Arabia, and the United Arab Emirates). ${ }^{21}$ Patients treated with an SU for a median duration of 4 years, with $(92 \%)$ or without ( $8 \%$ ) metformin, were either switched to sitagliptin $100 \mathrm{mg}$ once daily $(\mathrm{N}=529)$ or remained on their pre-study $\mathrm{SU}(\mathrm{N}=537)$ in addition to their previous metformin treatment where applicable. A total of 1021 of the 1066 randomized patients returned at least one completed diary card and were included in the analysis, 507 in the sitagliptin group and 514 in the SU group (35\% glibenclamide, 35\% glimepiride, and 30\% gliclazide prior to randomization). These patients had a mean age of 55 years, $51 \%$ were male, median duration of diabetes was 5.5 years, and mean $\mathrm{HbA}_{1 \mathrm{c}}$ at baseline was $7.5 \%$. For the Ramadan fasting period, patients were provided with daily diary cards to record hypoglycemia and were instructed to perform finger stick glucose measurements if they experienced symptoms. More than $90 \%$ of patients reported that they did not break the fast for reasons other than treatment of hypoglycemia.

The data further showed that the proportion of patients who recorded one or more symptomatic hypoglycemic events during Ramadan was lower in the sitagliptin group (6.7\%; 128 events in 34 patients) compared with the SU group $(13.2 \% ; 195$ events in 68 patients $)$, resulting in a risk ratio of $0.51(95 \% \mathrm{CI}, 0.34-0.75 ; P<0.001)$. The difference was driven by higher incidences of hypoglycemia reported with glibenclamide (19.7\%) and glimepiride (12.4\%), while the incidence with gliclazide $(6.6 \%)$ was very similar to that seen with sitagliptin $(6.7 \%)$. As reported by others, ${ }^{14}$ the incidence of symptomatic hypoglycemia in this study varied widely across countries (ranging in the SU group from $0.5 \%$ in Saudi Arabia to $26.2 \%$ in Israel). In addition, clear differences between the two groups were only seen in three (Egypt, Israel, Lebanon) of the six countries. Consistent data to those for symptomatic hypoglycemia were seen for the combination of symptomatic and asymptomatic (blood glucose $\leq 3.9 \mathrm{mmol} / \mathrm{L}$ [70 $\mathrm{mg} / \mathrm{dL}]$ ) hypoglycemic events $(8.5 \%$ versus $17.9 \%)$ and confirmed (blood glucose $\leq 3.9 \mathrm{mmol} / \mathrm{L}[70 \mathrm{mg} / \mathrm{dL}]$ ) symptomatic hypoglycemia ( $1.6 \%$ versus $4.3 \%$ ). No events that required medical assistance (ie, visits to the physician or emergency room or hospitalizations) or were considered severe were reported. However, a low percentage of patients $(0.2 \%$ in the sitagliptin group and $0.8 \%$ in the SU group) had events requiring nonmedical assistance. In this study, measures of glycemic control or weight were not assessed.

Other than hypoglycemia, 19 adverse events (AEs) were recorded: three patients reported a total of three AEs in the sitagliptin group, and nine patients reported a total of 16 AEs in the SU group, three of which were serious AEs (SAEs) (ischemic stroke, acute pancreatitis, and urinary tract infection).

A second, similar study evaluated sitagliptin in patients with T2DM fasting during Ramadan from Asia. ${ }^{22}$ This multicenter, randomized, open-label, pragmatic trial enrolled 870 patients from India $(\mathrm{N}=765)$ and Malaysia $(\mathrm{N}=105)$ treated with a stable dose of sulfonylurea with $(86 \%)$ or without concomitant metformin. Patients were randomized to either remain on their pre-study $\mathrm{SU}(\mathrm{N}=434$; median duration of SUs of 1 year; $65 \%$ glimepiride [median dose at baseline $2 \mathrm{mg} /$ day], 22\% glibenclamide [median dose $10 \mathrm{mg} /$ day], and $13 \%$ gliclazide [median dose $80 \mathrm{mg} /$ day]) or to switch to sitagliptin $100 \mathrm{mg}$ once daily $(\mathrm{N}=436)$, with (median dose $1,000 \mathrm{mg} /$ day) or without metformin. A total of 848 of the 870 randomized patients returned at least one completed diary card and were included in the analysis, 421 in the sitagliptin group and 427 in the SU group. Baseline characteristics included a mean age of 51 years, $47 \%$ male patients, a median duration of T2DM of 3 years, and a mean $\mathrm{HbA}_{1 \mathrm{c}}$ of $8.0 \%$. As for the previous study, daily diary cards were given to the patients to document information on hypoglycemic symptoms and complications, and patients were instructed to perform finger stick glucose measurements. Nearly all patients $(>97 \%)$ indicated that they did not break the fast for reasons other than 
treating hypoglycemia. Treatment changes of either dose or mostly timing occurred in $13.1 \%$ of sitagliptin-treated patients and $22.7 \%$ of SU-treated patients.

In line with the results from the previous study, fewer patients in the sitagliptin group $(3.8 \% ; 22$ events in 16 patients) recorded at least one symptomatic hypoglycemic event during Ramadan compared with the SU group (7.3\%; 63 events in 31 patients), resulting in a risk ratio of 0.52 (95\% CI, 0.29-0.94; $P=0.028)$. None of the events led to discontinuation. The results varied again among the different SUs, with the proportion of patients experiencing symptomatic events being $9.1 \%$ with glimepiride and $5.2 \%$ with glibenclamide but only $1.8 \%$, ie, even slightly lower than with sitagliptin (3.8\%), in the gliclazide group. Among the two participating countries, differences in the relative incidence of hypoglycemia between sitagliptin- and SU-treated patients were consistent, although overall lower rates were reported in Malaysian patients. Consistent data to those seen for symptomatic events were reported for the combination of symptomatic and asymptomatic (blood glucose $\leq 3.9 \mathrm{mmol} / \mathrm{L}$ [70 mg/dL]) hypoglycemic events (4.8\% versus $9.6 \%)$ and confirmed (blood glucose $\leq 3.9 \mathrm{mmol} / \mathrm{L}[70 \mathrm{mg} / \mathrm{dL}]$ ) symptomatic hypoglycemia $(2.1 \%$ versus $5.4 \%)$. None of the events in either group required medical assistance, and $0.2 \%$ of patients in the sitagliptin group and $1.6 \%$ in the SU group reported severe hypoglycemia requiring nonmedical assistance.

The proportion of patients experiencing AEs other than hypoglycemia was $10.0 \%$ in the sitagliptin group (including one SAE [car accident]) and $7.0 \%$ in the SU group, with five patients on sitagliptin and none on SUs discontinuing due to AEs.

Taken together, in both these studies, the incidence of symptomatic hypoglycemia during Ramadan was reduced by about a half with sitagliptin compared with SU treatment, with the exception of gliclazide therapy, which was associated with a lower incidence of hypoglycemia, similar to that of sitagliptin.

In addition to the specific trials with sitagliptin discussed above, a prospective, observational, cross-sectional study conducted in Saudi Arabia, which focused on extensive patient education for Ramadan, also included patients receiving sitagliptin or vildagliptin (combined as DPP-4 inhibitors). ${ }^{18}$ While only 42 out of the 1046 enrolled patients (92\% with T2DM) were treated with a DPP-4 inhibitor (in different combinations with oral antidiabetic drugs/ insulin), none of these patients experienced hypoglycemic episodes during Ramadan fasting. The overall percentage of patients experiencing hypoglycemia during Ramadan in this study was $4.6 \%$.

\section{Vildagliptin}

Vildagliptin was initially evaluated in patients with T2DM fasting during Ramadan in two studies conducted in the UK.

Devendra et al conducted an observational, nonrandomized, non-interventional study, collecting data from primary care practices in North West London. ${ }^{23}$ A total of 52 patients insufficiently controlled on metformin $2 \mathrm{~g}$ /day were given either vildagliptin $50 \mathrm{mg}$ twice daily (bid) $(\mathrm{N}=26)$ or gliclazide $(\mathrm{N}=26 ; 80 \mathrm{mg}$ bid, titrated to $160 \mathrm{mg}$ bid if fasting glucose was $>7 \mathrm{mmol} / \mathrm{L}$ on day 8 ) as add-on therapy prior to inclusion into the study. About two-thirds of these patients were female. In the vildagliptin group $46.2 \%$ of patients were from Middle Eastern origin and 38.5\% were Pakistani, with a mean age of 53 years, while $92.3 \%$ of gliclazide-treated patients were Pakistani, with a mean age of 62 years. Both groups had a similar mean diabetes duration ( 7 years) and $\mathrm{HbA}_{1 \mathrm{c}}(9 \%)$. Participants were instructed to monitor and record hypoglycemic episodes and were all provided with a glucose meter to measure blood glucose levels in case they experienced hypoglycemic events; both diaries and meters were analyzed.

The number of patients experiencing hypoglycemic events (defined as blood glucose $<3.5 \mathrm{mmol} / \mathrm{L}$ with or without symptoms) was markedly lower with vildagliptin (7.7\%; two patients experiencing two events) than gliclazide (61.5\%; 16 patients experiencing 24 events; $P<0.001)$. No severe hypoglycemic episodes (ie, needing assistance to treat) were reported in the vildagliptin group, and one such event was seen in the gliclazide group. $\mathrm{HbA}_{1 \mathrm{c}}$ reductions from pre- to post-Ramadan were comparable in both groups $(-1.26 \%$ with addition of vildagliptin to metformin and $-1.23 \%$ with addition of gliclazide to metformin; $P=0.822$ ). Weight changes over the same period were minimal (least-squares mean change of $+0.12 \mathrm{~kg}$ with vildagliptin and $+0.38 \mathrm{~kg}$ with gliclazide; $P=0.4709)$. No safety data other than hypoglycemia were reported.

The second Ramadan study conducted with vildagliptin in the UK was a prospective, observational, nonrandomized, openlabel, two-cohort study, called VECTOR (Vildagliptin Experience Compared To gliclazide Observed during Ramadan). ${ }^{24,25}$ Of the 72 patients enrolled, 30 patients had been prescribed vildagliptin (50 mg bid) add-on to metformin (free or fixed combination) and 41 patients SU add-on to metformin before study start and continued this treatment during the study; one patient was not allocated to treatment. ${ }^{24}$ The 59 patients who 
completed the study $(\mathrm{N}=23$ for the vildagliptin cohort and $\mathrm{N}=36$ for the gliclazide cohort) were included in the analysis. Discontinuations were due to loss of follow-up including no medication intake, no fasting or nonattendance of post-Ramadan visit. Nearly all patients (97\%) were South Asian and 56\% were male. Mean exposure to current metformin dose before fasting was 59.6 weeks in the vildagliptin group and 197.5 weeks in the SU group, respectively, and exposure to current vildagliptin and SU doses were 21.2 weeks and 57.0 weeks, respectively. All of the SU-treated patients received gliclazide (median dose $80 \mathrm{mg}$ / day), and the median dose of metformin was $2,000 \mathrm{mg}$ /day in both cohorts. Additional demographic characteristics included a mean age of 58 years and mean T2DM duration of 6.3 years. Baseline $\mathrm{HbA}_{1 \mathrm{c}}$ was somewhat higher in the vildagliptin cohort $(7.7 \%)$ than in the gliclazide cohort $(7.2 \%)$. Both cohorts fasted for a comparable number of days (25.0 versus 25.4 for vildagliptin and gliclazide, respectively). Dose adjustments for Ramadan occurred in $13.9 \%$ of SU-treated patients and no vildagliptin-treated patients.

During Ramadan, no patients $(0 \%)$ in the vildagliptin cohort reported hypoglycemic events (defined as patientreported symptoms and/or blood glucose $<3.9 \mathrm{mmol} / \mathrm{L}$ [70 mg/dL]) compared with $41.7 \%$ of patients (34 events in 15 patients; $P=0.0002$ ) treated with gliclazide, including one severe event (ie, requiring third-party assistance). In the subset of patients having both pre- and post-Ramadan assessments, $\mathrm{HbA}_{1 \mathrm{c}}$ decreased from $7.7 \%$ to $7.2 \%$ in the vildagliptin group ( $\mathrm{N}=20)$ and increased slightly from $7.2 \%$ to $7.3 \%$ in the SU group $(\mathrm{N}=32)$; the between-group difference $(-0.5 \%)$ was statistically significant $(P=0.0262)$. Bodyweight remained constant in both cohorts $(78.8 \mathrm{~kg}$ pre- and post-Ramadan with vildagliptin [N=22] and 76.4 and $76.8 \mathrm{~kg}$, respectively, with gliclazide $[\mathrm{N}=36]$ ). A better treatment adherence during Ramadan was found for vildagliptin-treated versus gliclazidetreated patients. ${ }^{25}$ In the vildagliptin group, one patient (4.3\%) missed a total of four doses, whereas in the gliclazide group, ten patients (27.8\%) missed a total of 266 doses. Expressed in mean proportion of doses missed during Ramadan, this was $0.2 \%$ with vildagliptin and $10.4 \%$ with the SU $(P=0.029)$.

AEs other than hypoglycemia were experienced by six patients in the SU cohort, with none of the events being serious, while no AEs were reported in the vildagliptin cohort. ${ }^{24}$

Another small, multicenter, non-interventional/ observational, nonrandomized, open-label study with vildagliptin was exclusively conducted in Indian Muslim patients with T2DM. ${ }^{26}$ At the time of finalization of this review, this study was published in abstract form only; however, it was still considered for completeness. A total of 97 patients taking vildagliptin $(\mathrm{N}=55)$ or SUs $(\mathrm{N}=42)$ as monotherapy or combination therapy with metformin were included in the study. Patients had a mean age of 51 years, with a mean $\mathrm{HbA}_{1 \mathrm{c}}$ of $8.75 \%$ (vildagliptin arm) and 8.64\% (SU arm), respectively. Fasting was observed for a period of 29 days.

Of the patients in the SU group, $4.8 \%$ experienced hypoglycemic events (defined as patient-reported symptoms with or without blood glucose $<3.9 \mathrm{mmol} / \mathrm{L}$ [70 mg/dL]) compared with no $(0 \%)$ patients in the vildagliptin group $(P=0.104$ for between-group difference). The relatively low incidence of hypoglycemia in the SU cohort in this study could be related to the high baseline $\mathrm{HbA}_{1 \mathrm{c}}$ and is also consistent with the observation in an earlier study in which investigators chose not to escalate the dose of the SU to avoid hypoglycemia. ${ }^{27}$ There was a significant decrease in $\mathrm{HbA}_{1 \mathrm{c}}$ from pre- to post-Ramadan in vildagliptin-treated patients $(-0.43 \% ; P=0.009)$, while $\mathrm{HbA}_{1 \mathrm{c}}$ remained unchanged in SU-treated patients $(+0.01 \%$; $P=0.958)$. In addition, bodyweight decreased by $-1.2 \mathrm{~kg}$ in the vildagliptin group and remained stable $(-0.03 \mathrm{~kg})$ in the $\mathrm{SU}$ group ( $P=0.011$ for between-group difference).

The VERDI (Vildagliptin Experience during Ramadan in patients with DIabetes) study was a multicenter, prospective, observational, nonrandomized, open-label study conducted in France. ${ }^{28}$ A total of 218 patients on oral dual-therapy of metformin-vildagliptin or metformin-sulphonylurea/glinide (insulin secretagogue [IS] cohort) were recruited ( $\mathrm{N}=115$ for vildagliptin and $\mathrm{N}=83$ for IS), and 198 patients were included in the analysis ( $\mathrm{N}=120$ for vildagliptin and $\mathrm{N}=78$ for IS). Of these patients $90 \%$ were from Maghreb, and mean age was 59 years, with $60 \%$ being male, mean T2DM duration of 7.4 years, mean metformin dose of $>2,100 \mathrm{mg} /$ day, and a mean baseline $\mathrm{HbA}_{1 \mathrm{c}}$ of $7.2 \%$. While information about the type of SUs used in the study was not collected, it is noteworthy that in France, the most widely prescribed SU, by far, is gliclazide. Depending on local practice, patients could be advised to specifically monitor their blood glucose levels (which occurred in $80.5 \%$ of IS-treated and $68.4 \%$ of vildagliptin-treated patients) and be provided with a glucometer; however, no study diary was requested to collect hypoglycemic events or record values of glucose monitoring. Patients fasted for an average of 29.8 days (range 16-31 days). Distinct therapeutic management was planned in view of Ramadan with drug-adaptation actually performed in $28.3 \%$ of patients in the vildagliptin group and $66.7 \%$ of patients in the IS group.

Categories of hypoglycemic episodes were defined in this study as symptomatic, confirmed (blood glucose measurement $<3.9 \mathrm{mmol} / \mathrm{L}[70 \mathrm{mg} / \mathrm{dL}]$ ), severe (need of third-party assistance), reported as AEs, or requiring an 
unscheduled medical visit. The overall frequency of any malaise suggestive of hypoglycemia was relatively high in both cohorts, reported in about one-third of patients, with a small between-group difference (37.2\% in the IS cohort and $34.2 \%$ in the vildagliptin cohort). This might be attributable to the much extended fasting hours of over 18 hours during summer days in France in the year 2012, making it more difficult to ascertain the hypoglycemic nature of all the malaises. However, the frequency of better-documented hypoglycemic episodes and/or more severe events was consistently lower under vildagliptin therapy compared with insulin-secretagogues. In particular, there was a higher incidence of hypoglycemic episodes documented by the physician as AEs in the IS cohort (17.9\% of patients reporting 22 events) than in patients treated with vildagliptin ( $7.5 \%$ of patients reporting 13 events; $P=0.025$ ). The percentage of patients reporting at least one severe episode of hypoglycemia that required third-party assistance was $3.9 \%$ in the IS cohort and $1.7 \%$ in the vildagliptin cohort. Of the patients in the IS cohort, $7.7 \%$ had at least one unscheduled medical visit during Ramadan because of hypoglycemia, compared with $1.7 \%$ in the vildagliptin cohort. Glycemic control (mean $\mathrm{HbA}_{1 \mathrm{c}}=7.2 \%$ in either cohort before and after Ramadan) and bodyweight $(-0.5 \mathrm{~kg}$ in the IS group and $-0.7 \mathrm{~kg}$ in the vildagliptin group) remained stable and similar in both cohorts during Ramadan.

AEs were reported by $7.5 \%$ of patients in the vildagliptin cohort and $19.2 \%$ of patients in the IS cohort, the difference being mainly driven by hypoglycemia as summarized above. The additional events reported were one case of hyperglycemia with vildagliptin and malaise, dizziness, and hyperhidrosis with IS. No events led to discontinuation or were reported as SAE.

The VIRTUE (VildagliptIn expeRience compared wiTh sulphonylUreas obsErved during Ramadan) study was a large multicenter, prospective, non-interventional, nonrandomized, open-label trial with vildagliptin conducted in ten countries in the Middle East (Lebanon, Egypt, UAE, Oman, Saudi Arabia, and Kuwait) and Asia (Pakistan, India, Bangladesh, and Indonesia). ${ }^{29}$ Of 1,333 patients enrolled, 684 were treated with vildagliptin and 631 were treated with an SU; 18 patients did not receive medication of interest and consequently were not included in subsequent analysis. Of the patients in either cohort, $98 \%$ completed the study. At the start of Ramadan, most patients received dual therapy with metformin, $90.7 \%$ in the vildagliptin cohort and $86.7 \%$ in the SU cohort (median dose of metformin 1,700 and 1,500 $\mathrm{mg} /$ day, respectively) and in the SU group, 55\% of patients received glimepiride (median dose $2 \mathrm{mg} /$ day), $31 \%$ gliclazide (median dose $60 \mathrm{mg} /$ day), $10 \%$ glibenclamide (median dose $5 \mathrm{mg} /$ day), and $1 \%$ glipizide (median dose $10 \mathrm{mg} /$ day). Demography and baseline characteristics further showed a mean age of 49.6 years, a mean duration of $\mathrm{T} 2 \mathrm{DM}$ of 3.9 years, and a mean $\mathrm{HbA}_{1 \mathrm{c}}$ of $7.3 \%$ (vildagliptin group) and 7.4\% (gliclazide group), respectively; $58.7 \%$ of the patients were male. Patients were provided with an optional diary for recording hypoglycemic symptoms and blood glucose levels, if measured. Patients fasted for a mean of 28.5 days in the vildagliptin cohort and 28.6 days in the SU cohort. Adaptations to study medication in preparation for Ramadan occurred in $9.9 \%$ of patients on vildagliptin and $20.2 \%$ of patients on an SU, and further medication changes during Ramadan were reported for only $1.8 \%$ of patients in the vildagliptin cohort and $4.6 \%$ of patients in the SU cohort. In addition, the median daily doses of the antidiabetic treatments were the same before and after Ramadan.

Significantly fewer patients reported hypoglycemic events (defined as any reported symptoms by the patient and/or any blood glucose measurement $<3.9 \mathrm{mmol} / \mathrm{L}$ [70 mg/dL]) with vildagliptin ( $\mathrm{N}=36 / 669 ; 5.4 \%)$ compared with SUs ( $\mathrm{N}=123 / 621 ; 19.8 \% ; P<0.001$ for between-group difference). An even larger difference was found when only considering events confirmed by a low blood glucose measurement $<3.9 \mathrm{mmol} / \mathrm{L}$. Of the vildagliptin-treated patients, $2.7 \%$ reported such an event, compared with $12.9 \%$ of SU-treated patients. None of the vildagliptin-treated patients experienced a severe hypoglycemic episode (ie, requiring third-party assistance), while this occurred in four patients on SUs $(P=0.053)$. The incidence of hypoglycemia was markedly lower with vildagliptin when compared with each individual $\mathrm{SU}$, for which the hypoglycemia rates were $17.9 \%$ for glimepiride, $19.2 \%$ for gliclazide, $31.8 \%$ for glibenclamide, and $12.5 \%$ for glipizide. The mean change in $\mathrm{HbA}_{1 \mathrm{c}}$ from pre-Ramadan to study end was $-0.24 \%$ in the vildagliptin group and $+0.02 \%$ in the SU group $(P<0.001$ for between-group difference). In addition, bodyweight changes were -0.76 and $-0.13 \mathrm{~kg}$, respectively $(P<0.001$ for betweengroup difference). Treatment adherence during Ramadan was good, with similarly low mean number of missed doses in either cohort ( 0.7 with vildagliptin versus 0.8 with SUs).

AEs were reported in $10.2 \%$ of vildagliptin-treated patients and $22.8 \%$ of SU-treated patients, with the difference driven by hypoglycemia, and all other AEs reported by $\leq 1.3 \%$ of patients. Seven patients experienced SAEs, 


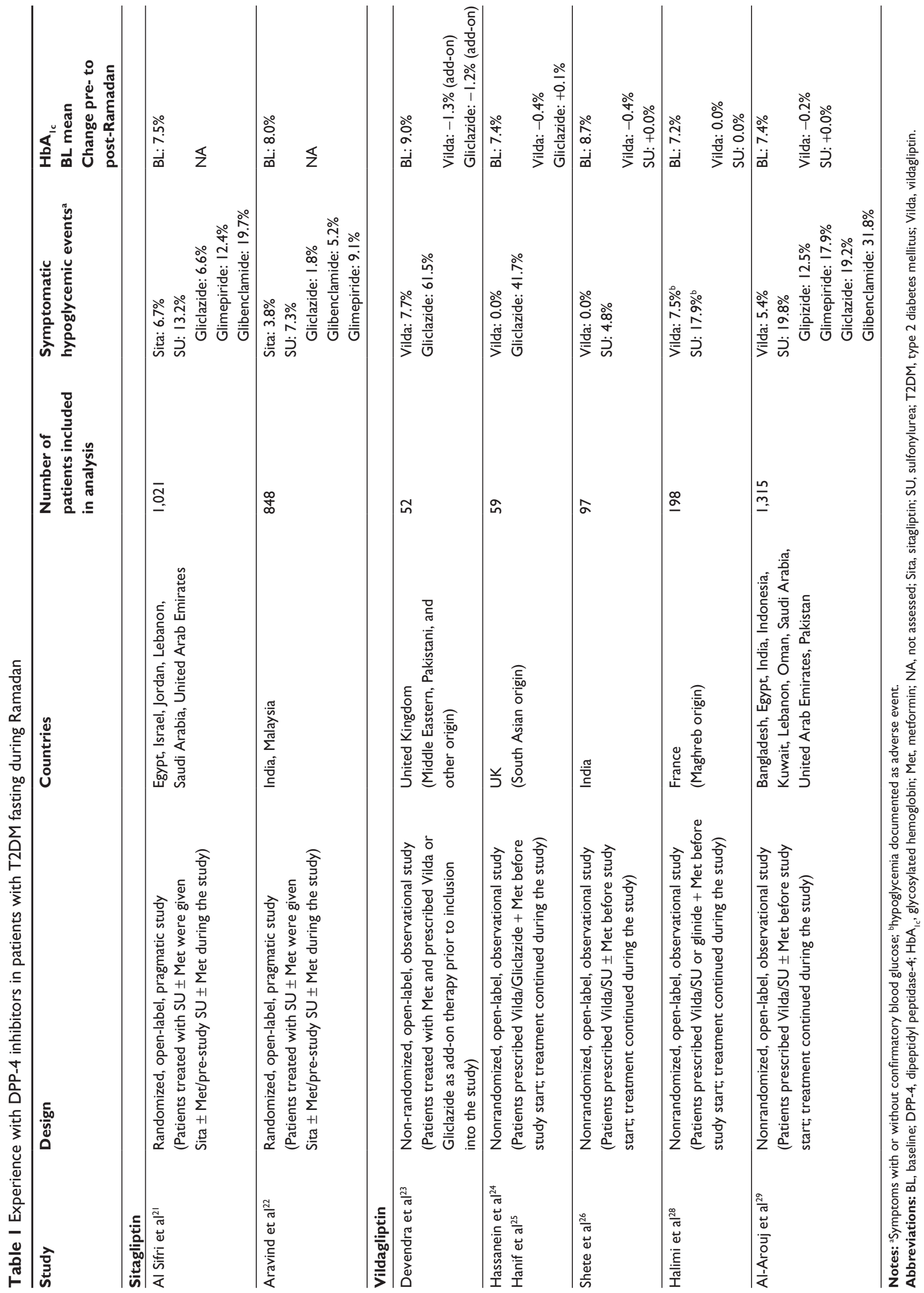


two in the vildagliptin cohort (one myocardial infarction and one viral hepatitis) and five in the SU cohort (one transient ischemic attack and four hypoglycemia).

Taken together, data is accruing with vildagliptin in patients with T2DM fasting during Ramadan from various parts of the world and under different types of diet (Table 1). Overall, they show a consistent effect of a lower frequency of hypoglycemia with vildagliptin versus SUs during Ramadan, including compared with the SU gliclazide.

\section{Mechanistic considerations}

Among the body's defense against hypoglycemia, following an initial reduction in insulin secretion as a first-line protection, glucagon plays a key role among the counter-regulatory factors. ${ }^{11}$ Thus, preserving this response is essential to minimizing the risk of hypoglycemia with any antidiabetic medication, especially given that even modestly advanced T2DM is already associated with an impaired glucagon response. ${ }^{30}$ This becomes even more important in certain patient groups who are at increased risk of hypoglycemia, including those fasting during Ramadan.

As described earlier, the incretin hormones GLP-1 and GIP increase the sensitivity of the $\alpha$ - and $\beta$-cell to glucose, resulting in glucose-dependent secretion of insulin and glucagon, which underlie their low propensity to elicit hypoglycemia. When glucose levels fall in the hypoglycemic range, this leads to a more rapid and pronounced decrease in insulin release and a more rapid and pronounced increase in glucagon. This preserved glucagon counter-regulation has specifically been shown for the DPP-4 inhibitor vildagliptin in placebo-controlled crossover studies both in diet- and insulin-treated patients with T2DM as well as in patients with T1DM. ${ }^{31-33}$ While vildagliptin suppresses the inappropriate glucagon secretion during hyperglycemia (as assessed during standard meals), it sustained or even enhanced the glucagon response to hypoglycemia $(2.5 \mathrm{mM}$ glucose clamp following the standard meals). The observed effect on glucagon is likely a GIP-medicated effect; GIP was recently shown by Christensen and coworkers to have a glucagonotropic effect during hypoglycemic conditions. ${ }^{34}$ An important mechanistic consideration is that for any GIP-mediated effect on glucagon to result in protection against hypoglycemia, the levels would need to remain elevated in the inter-meal and overnight periods, when hypoglycemia is most likely to occur, which has actually been shown for vildagliptin treatment, including an increase in fasting GLP-1 and GIP levels. ${ }^{19}$ A likely explanation for the extension of the meal-induced increases into the inter-meal and overnight periods is that - through the nature of interacting with the DPP-4 enzyme as a substrate-like blocker (rather than a competitive enzyme inhibitor) ${ }^{35}$ and its bid regimen - treatment with vildagliptin results in an essentially chronic blocking of the degradation of the incretin hormones. ${ }^{19}$

In contrast to the effects of DPP-4 inhibition on glucagon counter-regulation, SUs have been shown to significantly impair the glucagon response in T2DM patients. Both tolbutamide and glibenclamide, for example, have been shown to suppress the glucagon counter-regulation to hypoglycemia. ${ }^{36,37}$ A differential effect between an SU and a DPP-4 inhibitor on $\alpha$-cell function has also been shown in a 2-year study, in which vildagliptin reduced the meal-induced glucagon response in patients with T2DM who are treated with metformin, while glimepiride, over the same period, resulted in an increase in glucagon levels, which may be the result of attenuation of the glucose sensitivity of the $\alpha$-cells with uncoupling of glucose dependency. ${ }^{38}$ It is, however, important to bear in mind, as mentioned earlier, that the hypoglycemic potential of different SUs is not identical, and that gliclazide has consistently been associated with the lowest hypoglycemic incidence, both in the general T2DM population ${ }^{12}$ and during Ramadan. ${ }^{14}$ Glyburide, in particular, carries the highest risk, and recently has been added to the updated 2012 American Geriatric Society Beers Criteria for potentially inappropriate medication use in older adults because of its greater risk of severe, prolonged hypoglycemia. ${ }^{39}$

Overall, the DPP-4 inhibitor-associated preservation of the glucagon response to hypoglycemia largely contributes to explain the clinical benefit seen with these agents in patients exposed to a higher risk of hypoglycemia, such as during prolonged fasting.

\section{Conclusion}

Collectively, these results suggest that DPP-4 inhibitors, an antidiabetic therapy with a low propensity for hypoglycemia, could be a valuable strategy to more safely manage treatment of T2DM with OAD therapy during Ramadan or other religious events requiring fasting and/or major alterations in meal patterns, as can be seen in older people for example. The data so far have been obtained from observational/pragmatic studies, all bearing some methodological limitations while being close to real life, and it will therefore be of interest to expand the experience with DPP-4 inhibitors in fasting conditions, complementing the evidence from observational studies with that from randomized, double-blind, interventional clinical trials to further inform international guidelines. One such interventional trial is under way, investigating vildagliptin 
compared with gliclazide in patients with T2DM from Europe, the Middle East, and South East Asia fasting during Ramadan 2013 (ClinicalTrials.gov identifier: NCT01758380). ${ }^{40}$

\section{Acknowledgments}

The authors gratefully acknowledge Anuja Shah (Novartis Healthcare Pvt Ltd) for technical help.

\section{Disclosure}

Anja Schweizer and Sylvie Dejager are employed by Novartis and own shares. Serge Halimi has received fees for consultancy, advisory boards, speaking, clinical studies, travel or accommodation from Novo-Nordisk, Lilly, Sanofi, Novartis, Boehringer Ingelheim, MSD, BMS, Astra-Zeneca, and Roche. The authors have no other conflicts of interest in this work.

\section{References}

1. Pewforum.org [homepage on the Internet]. The future of the global Muslim population: projections for 2010-2030. Washington, DC: Pew Research Center; 2011. Available from: http://www.pewforum.org. Accessed August 28, 2013.

2. Idf.org [homepage on the Internet]. IDF Diabetes Atlas, 5th ed. Brussels, Belgium: International Diabetes Federation; 2011. Available from: http://www.idf.org/diabetesatlas. Accessed August 28, 2013.

3. Salti I, Benard E, Detournay B, et al. A population-based study of diabetes and its characteristics during the fasting month of Ramadan in 13 countries: results of the epidemiology of diabetes and Ramadan 1422/2001 (EPIDIAR) study. Diabetes Care. 2004;27(10):2306-2311.

4. Al-Arouj M, Assaad-Khalil S, Buse J, et al. Recommendations for management of diabetes during Ramadan: update 2010. Diabetes Care. 2010;33(8):1895-1902.

5. Fatima J, Karoli R, Chandra A, Naqvi N. Ramadan fasting in patients with type 2 diabetes mellitus: experience from a teaching hospital. Indian J Endocrinol Metab. 2012;16(2):323-324.

6. Almaatouq MA. Pharmacological approaches to the management of type 2 diabetes in fasting adults during Ramadan. Diabetes Metab Syndr Obes. 2012;5:109-119.

7. Rashed AH. The fast of Ramadan. BMJ. 1992;304(6826):521-522.

8. Amiel SA, Dixon T, Mann R, Jameson K. Hypoglycaemia in Type 2 diabetes. Diabet Med. 2008;25(3):245-254.

9. Ahmad J, Pathan MF, Jaleel MA, et al. Diabetic emergencies including hypoglycemia during Ramadan. Indian $J$ Endocrinol Metab. 2012;16(4):512-515

10. Loke SC, Rahim KF, Kanesvaran R, Wong TW. A prospective cohort study on the effect of various risk factors on hypoglycaemia in diabetics who fast during Ramadan. Med J Malaysia. 2010;65(1):3-6.

11. Ahrén B. Avoiding hypoglycemia: a key to success for glucoselowering therapy in type 2 diabetes. Vasc Health Risk Manag. 2013;9: $155-163$.

12. Schernthaner G, Grimaldi A, Di Mario U, et al. GUIDE study: double-blind comparison of once-daily gliclazide MR and glimepiride in type 2 diabetic patients. Eur J Clin Invest. 2004;34(8):535-542.

13. Tayek J. SUR receptor activity vs incidence of hypoglycaemia and cardiovascular mortality with sulphonylurea therapy for diabetics. Diabetes Obes Metab. 2008;10(11):1128-1129.

14. Aravind SR, Al Tayeb K, Ismail SB, et al. Hypoglycaemia in sulphonylurea-treated subjects with type 2 diabetes undergoing Ramadan fasting: a five-country observational study. Curr Med Res Opin. 2011;27(6):1237-1242.
15. Davidson MB. Recommendations for management of diabetes during Ramadan. Diabetes Care. 2006;29(3):745.

16. Elhadd TA, Al-Amoudi AA. Recommendations for management of diabetes during Ramadan. Diabetes Care. 2006;29(3):744-745.

17. Hui E, Devendra D. Diabetes and fasting during Ramadan. Diabetes Metab Res Rev. 2010;26(8):606-610.

18. Aziz KM. Effect of fasting ramadan in diabetes control status application of extensive diabetes education, serum creatinine with $\mathrm{HbA}_{1 \mathrm{c}}$ statistical ANOVA and regression models to prevent hypoglycemia. Recent Pat Endocr Metab Immune Drug Discov. 2013;7(3): 233-251.

19. Ahrén B, Schweizer A, Dejager S, Villhauer EB, Dunning BE, Foley JE. Mechanisms of action of the dipeptidyl peptidase-4 inhibitor vildagliptin in humans. Diabetes Obes Metab. 2011;13(9):775-783.

20. Dejager S, Schweizer A. Minimizing the risk of hypoglycemia with vildagliptin: Clinical experience, mechanistic basis, and importance in type 2 diabetes management. Diabetes Ther. 2011;2(2):51-66.

21. Al Sifri S, Basiounny A, Echtay A, et al. The incidence of hypoglycaemia in Muslim patients with type 2 diabetes treated with sitagliptin or a sulphonylurea during Ramadan: a randomised trial. Int J Clin Pract. 2011;65(1):1132-1140.

22. Aravind SR, Ismail SB, Balamurugan R, et al. Hypoglycemia in patients with type 2 diabetes from India and Malaysia treated with sitagliptin or a sulfonylurea during Ramadan: a randomized, pragmatic study. Curr Med Res Opin. 2012;28(8):1289-1296.

23. Devendra D, Gohel B, Bravis V, et al. Vildagliptin therapy and hypoglycaemia in Muslim type 2 diabetes patients during Ramadan. Int $J$ Clin Pract. 2009;63(10):1446-1450.

24. Hassanein M, Hanif W, Malik W, et al. Comparison of the dipeptidyl peptidase-4 inhibitor vildagliptin and the sulphonylurea gliclazide in combination with metformin, in Muslim patients with type 2 diabetes mellitus fasting during Ramadan: results of the VECTOR study. Curr Med Res Opin. 2011;27(7):1367-1374.

25. Hanif W, Malik W, Hassanein M, et al. Treatment adherence with vildagliptin compared to sulphonylurea as add-on to metformin in Muslim patients with type 2 diabetes mellitus fasting during Ramadan. Curr Med Res Opin. 2013;29(7):807-811.

26. Shete AVV, Indian Ramadan study group. Real life experience of usage of vildagliptin versus sulfonylurea therapy in fasting patients with type 2 diabetes during Ramadan: an Indian experience. Diabetologia. 2011; 54 Suppl 1:S342-S343.

27. Foley JE, Sreenan S. Efficacy and safety comparison between the DPP-4 inhibitor vildagliptin and the sulfonylurea gliclazide after two years of monotherapy in drug-naive patients with type 2 diabetes. Horm Metab Res. 2009;41(12):905-909.

28. Halimi S, Levy M, Huet D, Quere S, Dejager S. Experience with vildagliptin in type 2 diabetic patients fasting during ramadan in France: insights from the VERDI study. Diabetes Ther. Epub August 31, 2013.

29. Al-Arouj M, Hassoun AA, Medlej R, et al. The effect of vildagliptin relative to sulphonylureas in Muslim patients with type 2 diabetes fasting during Ramadan: the VIRTUE study. Int J Clin Pract. 2013;67(10): 957-963.

30. Israelian Z, Szoke E, Woerle J, et al. Multiple defects in counterregulation of hypoglycemia in modestly advanced type 2 diabetes mellitus. Metabolism. 2006;55(5):593-598.

31. Ahrén B, Schweizer A, Dejager S, et al. Vildagliptin enhances islet responsiveness to both hyper- and hypoglycemia in patients with type 2 diabetes. J Clin Endocrinol Metab. 2009;94(4):1236-1243.

32. Farngren J, Persson M, Schweizer A, Foley JE, Ahren B. Vildagliptin reduces glucagon during hyperglycemia and sustains glucagon counterregulation during hypoglycemia in type 1 diabetes. J Clin Endocrinol Metab. 2012;97(10):3799-3806.

33. Ahrén B, Farngren J, Persson M, Schweizer A, Foley J. Improved glucagon dynamics during hypoglycemia and food-rechallenge by DPP-4 inhibition by vildagliptin in insulin-treated patients with type 2 diabetes. Diabetologia. 2013;56 Suppl 1:S51. 
34. Christensen M, Vedtofte L, Holst JJ, Vilsboll T, Knop FK. Glucosedependent insulinotropic polypeptide: a bifunctional glucose-dependent regulator of glucagon and insulin secretion in humans. Diabetes. 2011;60(12):3103-3109.

35. Davis JA, Singh S, Sethi S, et al. Nature of action of Sitagliptin, the dipeptidyl peptidase-IV inhibitor in diabetic animals. Indian $J$ Pharmacol. 2010;42(4):229-233.

36. Landstedt-Hallin L, Adamson U, Lins PE. Oral glibenclamide suppresses glucagon secretion during insulin-induced hypoglycemia in patients with type 2 diabetes. J Clin Endocrinol Metab. 1999;84(9): 3140-3145.

37. Peacey SR, Rostami-Hodjegan A, George E, Tucker GT, Heller SR. The use of tolbutamide-induced hypoglycemia to examine the intraislet role of insulin in mediating glucagon release in normal humans. $J$ Clin Endocrinol Metab. 1997;82(5):1458-1461.
38. Ahrén B, Foley JE, Ferrannini E, et al. Changes in prandial glucagon levels after a 2-year treatment with vildagliptin or glimepiride in patients with type 2 diabetes inadequately controlled with metformin monotherapy. Diabetes Care. 2010;33(4):730-732.

39. American Geriatrics Society 2012 Beers Criteria Update Expert Panel. American Geriatrics Society updated Beers Criteria for potentially inappropriate medication use in older adults. JAm Geriatr Soc. 2012;60(4): 616-631.

40. Novartis Pharmaceuticals. Vildagliptin Compared to Gliclazide as Dual Therapy With Metformin in Muslim Patients With Type 2 Diabetes Fasting During Ramadan (STEADFAST). Available from: http:// clinicaltrials.gov/show/NCT01758380. NLM identifier: NCT01758380. Accessed November 25, 2013.
Vascular Health and Risk Management

\section{Publish your work in this journal}

Vascular Health and Risk Management is an international, peerreviewed journal of therapeutics and risk management, focusing on concise rapid reporting of clinical studies on the processes involved in the maintenance of vascular health; the monitoring, prevention and treatment of vascular disease and its sequelae; and the involvement of

\section{Dovepress}

metabolic disorders, particularly diabetes. This journal is indexed on PubMed Central and MedLine. The manuscript management system is completely online and includes a very quick and fair peer-review system, which is all easy to use. Visit http://www.dovepress.com/ testimonials.php to read real quotes from published authors. 